\title{
Nocodazole does not synchronize cells: implications for cell-cycle control and whole-culture synchronization
}

Received: 23 August 2005 / Accepted: 3 November 2005 / Published online: 24 January 2006

C) Springer-Verlag 2006

\begin{abstract}
It has been predicted that nocodazole-inhibited cells are not synchronized because nocodazole-arrested cells with a G2-phase amount of DNA would not have a narrow cell-size range reflecting the cell size of some specific, presumably G2-phase, cell-cycle age. Size measurements of nocodazole-inhibited cells now fully confirm this prediction. Further, release from nocodazole inhibition does not produce cells that move through the cell cycle mimicking the passage of normal unperturbed cells through the cell cycle. Nocodazole, an archetypal whole-culture synchronization method, can inhibit growth to produce cells with a G2-phase amount of DNA, but such cells are not synchronized. Cells produced by a selective (i.e., non-whole-culture) method not only have a specific DNA content, but also have a narrow size distribution. The current view of cell-cycle control that is based on methods that are not suitable for cell-cycle analysis must therefore be reconsidered when results are based on whole-culture synchronization.
\end{abstract}

Keywords Nocodazole - Synchronization - Cell cycle · G2 phase $\cdot$ Membrane elution $\cdot$ Mouse (leukemic cell line)

\section{Introduction}

In order to understand the passage of a cell through the cell cycle - from birth by division of a mother cell to the next

This work was supported by the National Science Foundation (grant MCB-0323346) and (in part) by the National Institutes of Health (University of Michigan's Cancer Center, support grant 5 P30 CA46592). G.I., M.T., and P. B. are associated with the

Undergraduate Research Opportunity Program of the University of Michigan, which also supported this research.

S. Cooper $(\bowtie) \cdot$ G. Iyer

M. Tarquini - P. Bissett

Department of Microbiology and Immunology,

University of Michigan Medical School,

Ann Arbor, MI 48109-0620, USA

e-mail: cooper@umich.edu

Tel.: +734-764-4215

Fax: +734-764-3562 division - it is important to know what events take place at different times during the cell cycle. Because the measurement of chemical events is difficult in single cells, a large amount of effort has been expended on methods to synchronize cells. A properly or well synchronized culture is one in which the cells move as a uniform cohort through the cell cycle and accurately reflect the events occurring to a growing unperturbed cell during passage through the cell cycle. The sine qua non of synchronization is that the cells move uniformly through the cell cycle and divide synchronously over a relatively narrow span of time (Cooper 2004a; Cooper and Shedden 2003).

At the outset, it is extremely important to distinguish between cells that are "aligned" for a particular property but not synchronized, and those cells that are truly synchronized. This distinction has sometimes been obscured by discussion of the cell in terms of two cycles, the "chromatin cycle" (the replication of DNA and the associated pattern within the cell cycle) and the "growth cycle" (the pattern of growth of cell mass and related cellular elements). Others have observed (Gong et al. 1995) that the cells that are "chemically" treated exhibit growth imbalance and higher heterogeneity with respect to all parameters measured, including the expression of cyclins and the degree of $\mathrm{pRB}$ phosphorylation. What is lacking in previous discussions of synchronization methodology is the generality of the rule that whole-culture methods cannot synchronize cells. A whole-culture method is a method whereby all of the cells in a culture are treated, and no cells are discarded, so that the entire population has been transformed into a synchronized cohort. This rule does not imply that whole-culture methods synchronize cells poorly, or weakly, or occasionally, but rather that such methods do not synchronize cells at all, even though the cells may be aligned for some particular property, such as DNA content.

The importance of this proposal, when put forward in a constructive way (i.e., nocodazole does not synchronize cells), is that methods that are widely used to study the cell cycle do not yield information regarding the normal cell cycle. Results of cyclic changes and other changes following whole-culture synchronization must perforce be 
artifacts of the treatment and not related to the normal unperturbed cell cycle.

Synchronization methods can be divided into two classes: whole-culture methods and selective methods (Cooper 1991). Whole-culture methods are those in which the identical treatment of all cells in a culture is proposed to lead to a group of cells that are arrested at a specific point in the cell cycle. Release of these growth-arrested cells is presumed to produce a synchronized culture. Selective methods are those methods in which a subset of the original population is selected to produce a synchronized culture, and the remaining cells are discarded.

Theoretical analysis has led to the conclusion that whole-culture methods cannot synchronize cells (Cooper 1998, 2003, 2004a,b; Cooper and Shedden 2003). Whereas a whole-culture method may produce cells with some particular common property (e.g., all cells have a uniform DNA content), the cell-size distribution produced after growth arrest does not reflect or mimic the cell size of cells of some particular cell-cycle age. Specifically, the cell-size distribution after a whole-culture synchronization method is predicted to be as wide as that of the original culture (Cooper 2003), whereas the cells of a truly synchronized culture should have a narrow size distribution reflecting the cell size of a normal cell at a particular cell age between birth and division.

A study of lovastatin, a compound that was originally proposed to be a general whole-culture synchronizing agent (Keyomarsi et al. 1991), has demonstrated that lovastatin does not synchronize cells. Time-lapse analysis of cell division following lovastatin arrest has shown that the cells do not divide synchronously (Cooper 2002). Furthermore, analysis of the published data showing synchronized S phases and arrest with a G1-phase amount of DNA has indeed indicated that $\mathrm{S}$ phases are not synchronized, and that the cells are not arrested with a G1phase amount of DNA (Cooper 2002).

Nocodazole is widely used as a synchronizing agent (see, for example, Harper 2005a; Jansen-Durr et al. 1993; Ludlow et al. 1993; Ouyang et al. 1998). Cells treated with nocodazole are arrested at or prior to mitosis. Support for this proposal is the widely reported observation that nocodazole treatment produces cells with a G2-phase amount of DNA (Kung et al. 1990). We now describe experiments measuring the size distribution of cells treated with nocodazole and the pattern of growth following release from growth arrest by nocodazole treatment. Comparison of DNA and size measurements of nocodazole-arrested cells with cells produced by a selective method (membrane elution) support the proposal that nocodazole-arrested cells are not synchronized.

Most importantly for our general understanding of the normal mammalian cell cycle, we argue that the results presented here are not restricted to the cells and conditions used in this study, but that they apply broadly and generally to other mammalian cells and other growth systems. The prohibition against the experimental demonstration of a universal negative, viz., that whole-culture methods cannot synchronize cells, does not apply in this case. This is because the experiments presented here are strongly buttressed by theoretical considerations rigorously supporting the proposal that whole-culture methods cannot synchronize cells. Here, to the theoretical concept that these methods "cannot synchronize cells", we add experimental results supporting the proposal that such methods "do not synchronize cells".

\section{Materials and methods}

\section{Chemicals}

Nocodazole was purchased from Acros Organics. A nocodazole stock solution in dimelthysulfoxide (DMSO, $10 \mathrm{mg} / \mathrm{ml}$ ) was prepared. Dilution of the stock solution into medium produced the final nocodazole concentrations. Control treatments with DMSO indicated that DMSO at the concentrations used to introduce nocodazole to the cells did not alter cell growth or the cell-phase distribution.

\section{Cells}

L1210 cells, a mouse leukemic line (ATCC designation CCL219) was used for all experiments. These cells are non-adherent and grow with a doubling time of approximately 9-10 h.

\section{Growth media}

Liebovitz's L-15 medium (cellgro by Mediatech, Herndon, VA 20171) was supplemented with $2 \mathrm{mg} / \mathrm{ml}$ glucose, $100 \mathrm{U} / \mathrm{ml}$ penicillin, $100 \mu \mathrm{g} / \mathrm{ml}$ streptomycin, and $10 \%$ cosmic calf serum (Hyclone; a modified calf serum substituting for fetal bovine serum). Cells were incubated at $37^{\circ} \mathrm{C}$ in sealed flasks, as $\mathrm{L}-15$ medium does not require a $\mathrm{CO}_{2}$ atmosphere. Cells were never grown to above 200,000 cells $/ \mathrm{ml}$ in all experiments. Under these growth conditions, the cells were in steady-state growth and did not approach overgrowth conditions.

\section{DNA analysis}

Cells were collected by centrifugation, washed once in phosphate-buffered saline (PBS, pH 7.4), and suspended in $70 \%$ ethanol. After ethanol treatment in the cold for at least $20 \mathrm{~min}$, the cells were collected by centrifugation, the ethanol was aspirated off, and the cells were suspended in PBS containing propidium iodide $(50 \mu \mathrm{g} / \mathrm{ml})$ and RNase A $(100 \mu \mathrm{g} / \mathrm{ml})$. After at least $20 \mathrm{~min}$ incubation on ice, the cells were analyzed in a Becton-Dickinson FACScan analyzer by using Cellquest software. Further analysis of the data was carried out with WINMdi software. 


\section{Cell-size determinations}

Cells were counted and sized by using a Coulter Z2 electronic cell sizer and cell counter with a $70-\mu \mathrm{m}$ orifice. The data were collected and analyzed with the Z2 AccuComp program from Beckman Coulter (version 3.01). Cells were counted directly in $\mathrm{L}-15$ medium. Further analyses of the size distributions were performed by using Excel.

\section{Membrane elution}

The method has been described in detail previously (Eward et al. 2004; Helmstetter et al. 2003; Thornton et al. 2002) but will be briefly presented here. Cells are grown to a concentration of less than 200,000 cells $/ \mathrm{ml}$ to obtain approximately 60-70 million cells (e.g., $600 \mathrm{ml}$ cells at 100,000 cells $/ \mathrm{ml}$ contains 60 million cells). The entire experiment is carried out in a warm room. A membrane holder is placed in a full-view incubator within the warm room to ensure constant temperature. To start the production of newborn cells, $50 \mathrm{ml}$ warm $\left(37^{\circ} \mathrm{C}\right)$ PBS with $10 \mu \mathrm{g} / \mathrm{ml}$ concanavalin $\mathrm{A}$ is filtered through the membrane. Upon completion of filtration, no residual liquid remains. Then $100 \mathrm{ml}$ warm PBS is filtered through the membrane to remove unbound concanavalin A. Cells in 300-600 ml medium (containing 60-70 million cells) are subsequently filtered slowly onto the membrane with gentle suction over approximately 3-5 $\mathrm{min}$. When approximately $20-30 \mathrm{ml}$ liquid remains above the membrane, the liquid is poured off so that the cells never dry out or are exposed to air. The membrane apparatus is then inverted and filled with fresh medium, which is pumped through the membrane for 5-10 min, to remove non-bound cells. The cells obtained from this initial flow of medium through the membrane (together with the cells in the residual medium) are collectively referred to as the "wash-off", which is usually in the order of $10 \%-20 \%$ of the input cells. Thus, over $80 \%$ of the cells are bound to the membrane. After the wash-off is collected, the membrane is placed over a large funnel connected by tubing to a peristaltic pump. Fresh medium is pumped into the membrane holder at a rate of approximately $3.0 \mathrm{ml} / \mathrm{min}$. The pump connected to the bottom of the funnel pumps liquid at approximately $4.0 \mathrm{ml} / \mathrm{min}$. This prevents any collection of cells in the bottom of the funnel. Newborn cells for analysis are collected for 15-20 min.

\section{Results}

Size distribution of nocodazole-inhibited cells

Nocodazole was added to exponentially growing L1210 cells at various concentrations between 1 and $0.01 \mu \mathrm{g} / \mathrm{ml}$. After $19 \mathrm{~h}$, the size distributions and DNA distributions in the arrested cells were determined. Nocodazole-arrested cells were larger than uninhibited cells and had a wide size distribution (Fig. 1a). The results in Fig. 1a are shown in Fig. $1 \mathrm{~b}$ but with the size scale adjusted to compare the size
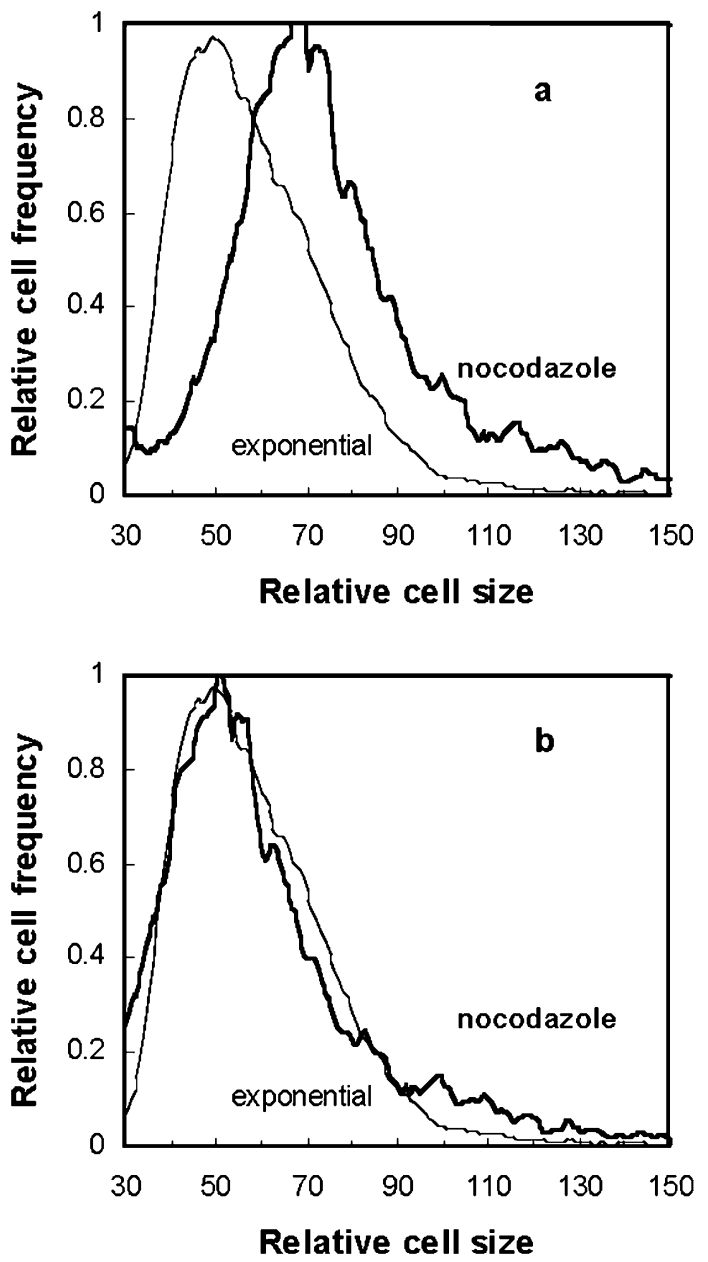

Fig. 1 Size distribution of nocodazole-inhibited cells compared with exponential control cells. L1210 cells were treated with nocodazole $(1.0 \mu \mathrm{g} / \mathrm{ml})$ for $19 \mathrm{~h}$. The cell size distribution of the inhibited cells was compared with untreated control cells. a Size distributions showing initial recorded absolute sizes. b The nocodazole size distribution was re-plotted to fit the peaks of the size distribution in the exponential distribution

distributions of the nocodazole-inhibited cells with exponential cells. The adjustment moves the peaks of the nocodazole-inhibited cells to coincide with the peak of the exponential cells. The breadth of the size distribution of the nocodazole-inhibited cells is essentially the same as the original cells.

\section{Size distribution of selection-synchronized cells}

A comparison of the nocodazole results with the production of newborn cells by membrane elution (a selective method that produces newborn cells without any inhibition, starvation, or perturbing treatments) is instructive. The classical membrane-elution method developed for bacteria (Helmstetter and Cummings 1963, 1964) has been adapted for eukaryotic cells by Helmstetter and colleagues (Eward et al. 2004; Helmstetter 1991; Helmstetter et al. 2003; Thornton et al. 2002). This eukaryotic membrane-elution method, as with the bacterial system, produces newborn 
eukaryotic cells continuously from cells bound to the membrane.

Figure 2 compares the absolute size distributions of newborn, exponential, and nocodazole-inhibited cells. Of note, the newborn cells have a size consistent with their being a subset of the exponential cells, but the nocodazoleinhibited cells are larger than the largest exponential cells.

DNA content of nocodazole-inhibited cells

Cells treated with nocodazole have a DNA content that is primarily comparable with that of G2 phase (Fig. 3). Thus, under conditions involving the arrest of cells with a G2phase amount of DNA, the inhibited cells do not reflect the sizes of cells seen during the normal G2 phase of the cell cycle (cf. Fig. 1).

Changes following release from nocodazole inhibition

When nocodazole-inhibited cells are washed to remove the nocodazole and then suspended in normal medium, the results are variable and highly dependent on the precise nocodazole concentration. One common result is illustrated in Fig. 4 in which cells produced by selective synchronization are compared with nocodazole-treated cells with respect to the ability of the cells to progress from having a G2-DNA content to having a G1-phase DNA content. In Fig. 4a (top line), the cells obtained by membrane elution are just entering the G2 phase of the cell cycle. Each subsequent line is the DNA content measured every hour. The cells leave the phase with a G2-DNA content by dividing to produce cells all having a G1-phase amount of DNA. In contrast, Fig. 4b shows cells arrested with a G2phase amount of DNA after nocodazole treatment. Upon removal of nocodazole, the cells are not able to divide to

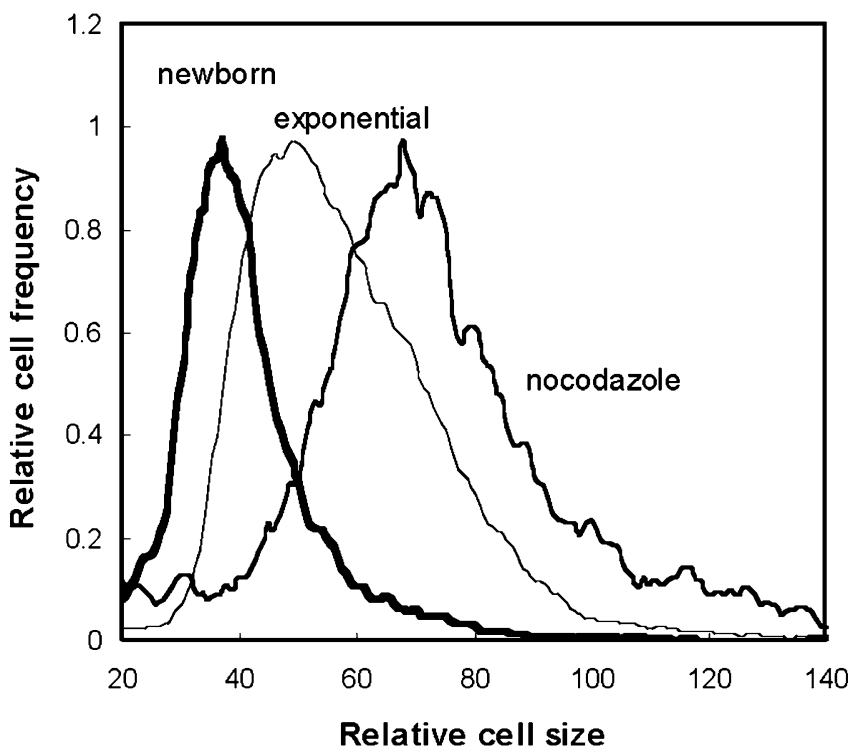

Fig. 2 Comparison of the absolute size variation of newborn, exponential, and nocodazole-inhibited cells

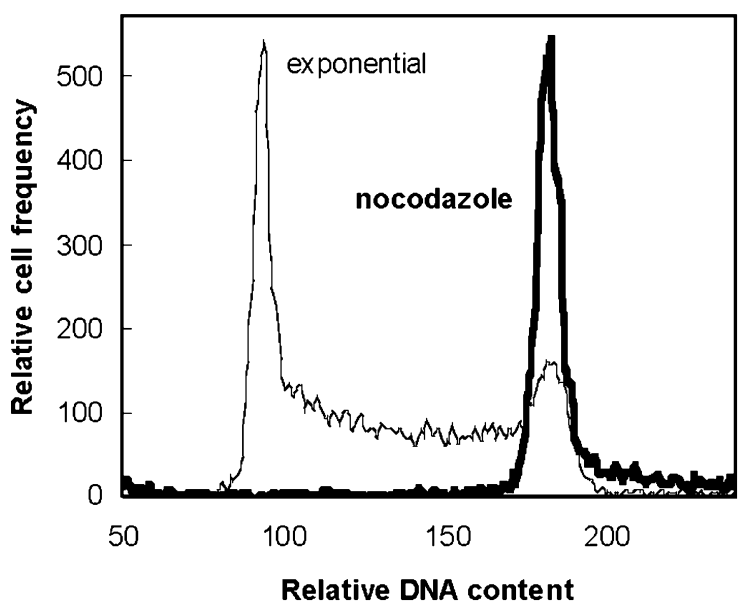

Fig. 3 DNA content of nocodazole-inhibited cells compared with DNA distribution of exponential cells

produce cells with a G1-phase amount of DNA. Whereas the cells obtained by membrane elution divide completely to produce cells with a G1-phase amount of DNA, the nocodazole-inhibited cells exhibit some division but then appear to "freeze" and do not continue to divide to produce cells with a G1-phase amount of DNA. Thus, the nocodazole-inhibited cells seem to be damaged to such an extent that they do not proceed normally through the cell cycle.
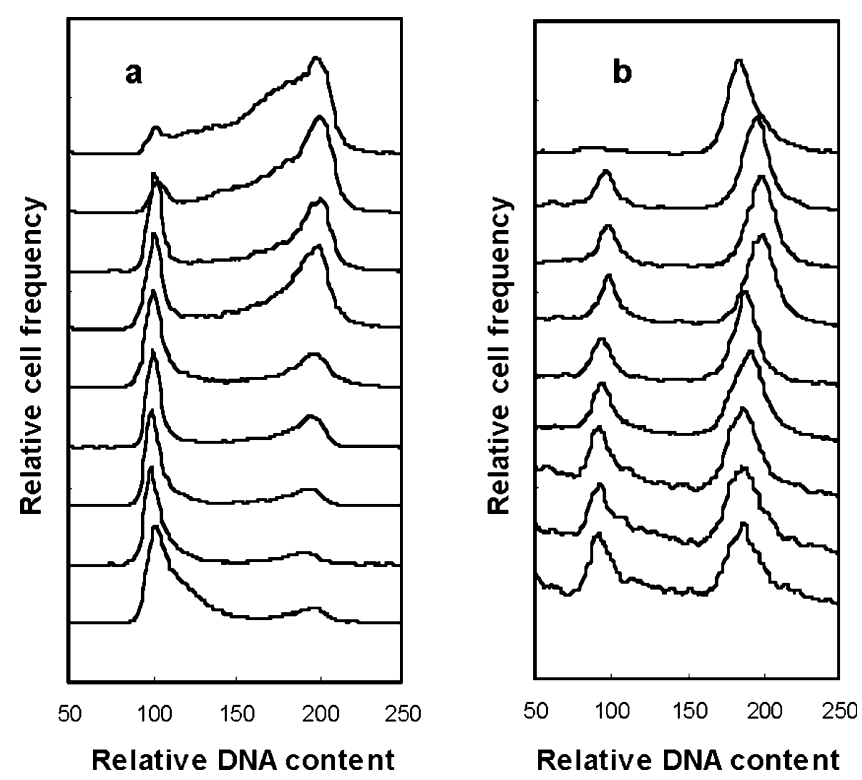

Fig. 4 Comparison of membrane-eluted cells and nocodazoleinhibited cells with respect to cells leaving the phase with G2-DNA content. a Membrane-eluted cells grown to produce cells with a G2 amount of DNA are shown at hourly intervals for comparison (time: top to bottom. All cells with a G2 amount of DNA lose this by division and produce cells with a G1-phase amount of DNA. b Nocodazole-inhibited cells (top line) were washed and restored to control medium. At hourly intervals, samples were taken, and the DNA content determined by fluorescence-activated chromosome flow-sorting. A fraction of the initial cells (top line) produce cells with a G1-phase amount of DNA, but not all cells aappear able to leave the G2-DNA state 


\section{Discussion}

"Synchronization" with nocodazole

The results reported here indicate that, although nocodazole inhibits cells to produce a cell population with a G2phase amount of DNA, these cells are not synchronized. Not only is the cell-size distribution of inhibited cells not narrowed as expected for a truly synchronized culture, but also, upon release of cells from nocodazole, the cells do not progress normally through the cell cycle. Thus, nocodazole has a deleterious or damaging (see Fig. 4) effect on cells. Over a range of concentrations, nocodazole produces effects that are inconsistent with cell synchronization. Most importantly, the nocodazole-inhibition experiments support the theoretical predictions that whole-culture methods cannot synchronize cells (Cooper 1998, 2004a,b; Cooper and Shedden 2003).

\section{Purpose of synchronization}

The purpose of synchronizing cells is to produce cells that can be used to measure and understand events occurring during the normal unperturbed cell cycle. A truly synchronized culture is one that mimics the passage of cells through the normal cell cycle. A newborn cell has a number of different properties (e.g., DNA content, cell size, protein composition, internal cellular arrangement), and as a cell passes through the cell cycle, each of these must necessarily change in order to produce a dividing cell at the end of the cell cycle. At a minimum, each component of the cell must double so that the pre-division cell has twice as much of everything that is present in the newborn cell. At each point during the cell cycle, a cell has a particular constellation of properties that are on the trajectory from newborn cell to dividing cell. A synchronized culture should, at each point during passage through the cell cycle, have cells with the specific properties associated with one particular cell-cycle age during the growth of an unperturbed cell.

If we consider two properties of a growing cell, e.g., cell size and DNA content, a newborn cell of age 0.0 has a size of 1.0 and a DNA content of the G1-phase. Size increases continuously during the cell cycle so that at division (age 1.0 ), the cell size is 2.0. DNA also increases during the cell cycle so that dividing cells have a DNA content that reflects the G2-phase amount of DNA. Just prior to division, e.g., at age 0.8 , a cell may have a G2-phase amount of DNA, and the cell size should be approximately 1.8. If a synchronized culture truly reflects the normal cell cycle, one would expect to find that cells arrested with a G2-phase amount of DNA have a relatively narrow cellsize distribution of about size 1.8. With biological and statistical variation considered, the size distribution could vary from 1.7 to 1.9 , or even be as wide as 1.6 to 2.0 . Nevertheless, the size distribution would be expected to be narrower than the size distribution in the original culture. If the size distribution is not narrowed, such a cell-size distribution would indicate that the cells are not arrested at a particular cell-cycle age reflecting the normal cell size at a point during the division cycle. Rather, such cells are presumably arrested with only one particular property common to all cells. In the case of nocodazole arrest, this property is a G2-phase amount of DNA. Other properties would be reflective of cells of all ages, as the size distribution in this case would include cells of all different cell-cycle sizes.

\section{Criteria for synchronization}

Several criteria have been proposed for recognizing a truly synchronized culture (Cooper 2004a; Cooper and Shedden 2003). From the list of 11 proposed criteria, those that are relevant to the experimental analysis are as follows:

1) If newborn cells are produced by the synchronization method, there should be a minimal increase in cell number for a period of time covering a significant fraction of the interdivision time.

2) The DNA distribution of cells should be narrow in the synchronized cells, and these distributions should then reflect the movement of cells through the division cycle. Thus, newborn cells should all have a G1-phase amount of DNA. The DNA content should then move through the S-phase content to a period of time when cells have only a G2-phase DNA content, followed by a return to an essentially pure G1-phase DNA content.

3) The size distribution of newly synchronized cells should be narrower than the size distribution of the original population, cell size should increase as the cells move through the cell cycle, and during the period of cell division, there should be a bi-modal distribution of cell sizes.

A more general consideration of the problem of nocodazole or whole-culture synchronization is that the alignment of cells so that all cells have a particular property in common (e.g. all cells have a G1-phase DNA content or a G2-phase DNA content) does not mean that the cells are synchronized. Synchronized divisions are the sine qua non of synchrony.

Application of these criteria to nocodazole treatment suggests that nocodazole-inhibited cells, according to our experimental results, are not synchronized. The cell-size distribution is not narrow, the cells do not move through the division cycle with a DNA content pattern indicative of normal cell-cycle passage, and there is no indication that cells divide synchronously.

Published work on synchronization

following nocodazole treatment

The literature on nocodazole use is enormous. Even the subset dealing only with the cell cycle is extremely large. We cannot re-analyze each and every paper that states that nocodazole synchronizes cells, but a general conclusion can be derived from those instances in which cells have been analyzed after release from nocodazole. The overwhelming 
result is that, after nocodazole is removed, cells do not fit the criteria of a synchronized culture. For example, Whitfield et al. (2002) produced cells that, following release from nocodazole treatment, did not have a pattern of DNA that indicated the cells were synchronized. Avisar et al. (2005) used nocodazole to "synchronize" cells, but no evidence for synchronization was presented. Another group used nocodazole to aid synchronization but noted that the resulting cells were not well synchronized (Summers et al. 2005), supporting the results reported here. A review of the use of nocodazole to synchronize cells has been published elsewhere (Harper 2005b).

When is the use of nocodazole valid?

Although we suggest that nocodazole does not synchronize cells, we do not wish to propose that the use of nocodazole to study aspects of cell biology is incorrect or that it represents bad science. Nocodazole may have its proper uses. A perusal of the literature on nocodazole indicates that a large number of studies with nocodazole are related to investigating the specific effects of nocodazole on cellular and biochemical phenomena. Thus, a study of the way that nocodazole inhibits microtubule formation and inhibits various cellular functions is valid. However, a suggestion that such studies are relevant to the events in the normal cell cycle would be invalid. The major problem is the overinterpretation of nocodazole-inhibition results in terms of cell-cycle studies. Our results suggest that those experiments that have involved the use of nocodazole to study the cell cycle through "nocodazole-induced synchronization" should be removed from the canon of accepted cell-cycle experiments.

Generalization of results to other cell lines and growth conditions

One critique of the possible extension of the results presented here to other cells and growth conditions might be that our results are merely related to the particular cells and growth conditions that we have used, viz., L1210 cells grown in L15 medium with cosmic calf serum (the cells and conditions used here), do not allow nocodazole to produce a synchronized culture. However, this argument ignores the theoretical generalization that led to the experiments presented here. Theory predicts the results presented here and that these results are independent of cell type or cell line.

Rather than placing the burden of proof on the proposal that all cell lines cannot be synchronized by whole-culture methods, we now shift the burden of proof to those who propose using such whole-culture methods, including nocodazole inhibition, as synchronization methods. We reject the argument proposing that perhaps "just around the corner" there exists a cell line or a cell situation that can be synchronized by such whole-culture methods. Theory predicts (Cooper 2003) that whole-culture synchronization cannot work, and the experiments presented here support this theory.

\section{References}

Avisar D, Segal M, Sneh B, Zilberstein A (2005) Cell-cycledependent resistance to Bacillus thuringiensis Cry1C toxin in Sf9 cells. J Cell Sci 118:3163-3171

Cooper S (1991) Bacterial growth and division. Academic Press, San Diego

Cooper S (1998) Mammalian cells are not synchronized in G1-phase by starvation or inhibition: considerations of the fundamental concept of G1-phase synchronization. Cell Prolif 31:9-16

Cooper S (2002) Reappraisal of G1-phase arrest and synchronization by lovastatin. Cell Biol Int 26:715-727

Cooper S (2003) Rethinking synchronization of mammalian cells for cell-cycle analysis. Cell Mol Life Sci 6:1099-1106

Cooper S (2004a) Is whole-culture synchronization biology's "perpetual motion machine"? Trends Biotech 26:266-269

Cooper S (2004b) Whole-culture synchronization can not, and does not, synchronize cells. Trends Biotech 22:274-276

Cooper S, Shedden K (2003) Microarray analysis of gene expression during the cell cycle. Cell Chromosom 2:1-12

Eward KL, Van Ert MN, Thornton M, Helmstetter CE (2004) Cyclin mRNA stability does not vary during the cell cycle. Cell Cycle 3:1057-1061

Gong J, Traganos F, Darzynkiewicz Z (1995) Growth imbalance and altered expression of cyclins B1, A, E, and D3 in MOLT- 4 cells synchronized in the cell cycle by inhibitors of DNA replication. Cell Growth Differ 6:1485-1493

Harper JV (2005a) Synchronization of cell populations in G1/S and G2/M phases of the cell cycle. Methods Mol Biol 296:157-166

Harper JV (2005b) Synchronization of cell populations in G1/S and G2/M phases of the cell cycle. Methods Mol Biol 296:157-166

Helmstetter CE (1991) Description of a baby machine for Saccharomyces cerevisiae. New Biol 3:1089-1096

Helmstetter C, Cummings D (1963) Bacterial synchronization by selection of cells at division. Proc Natl Acad Sci USA 50:767-774

Helmstetter C, Cummings D (1964) An improved method for the selection of bacterial cells at division. Biochim Biophys Acta 82:608-610

Helmstetter CE, Thornton M, Romero A, Eward KL (2003) Synchrony in human, mouse and bacterial cell culturesa comparison. Cell Cycle 2:42-45

Jansen-Durr P, Meichle A, Steiner P, Pagano M, Finke K, Botz J, Wessbecher J, Draetta G, Eilers M (1993) Differential modulation of cyclin gene expression by MYC. Proc Natl Acad Sci USA 90:3685-3689

Keyomarsi K, Sandoval L, Band V, Pardee AB (1991) Synchronization of tumor and normal cells from G1 to multiple cell cycles by lovastatin. Cancer Res 51:3602-3609

Kung AL, Sherwood SW, Schimke RT (1990) Cell line-specific differences in the control of cell cycle progression in the absence of mitosis. Proc Natl Acad Sci USA 87:9553-9557

Ludlow JW, Glendening CL, Livingston DM, DeCaprio JA (1993) Specific enzymatic dephosphorylation of the retinoblastoma protein. Mol Cell Biol 13:367-372

Ouyang B, Lan Z, Meadows J, Pan H, Fukasawa K, Li W, Dai W (1998) Human Bub1: a putative spindle checkpoint kinase closely linked to cell proliferation. Cell Growth Differ 9:877-885

Summers MK, Bothos J, Halazonetis TD (2005) The CHFR mitotic checkpoint protein delays cell cycle progression by excluding cyclin B1 from the nucleus. Oncogene 24:2589-2598

Thornton M, Eward KL, Helmstetter CE (2002) Production of minimally disturbed synchronous cultures of hematopoietic cells. Biotechniques 32:1098-1105

Whitfield M, Sherlock G, Saldanha A, Murray JI, Ball CA, Alexnder KE, Matese JC, Perou CM, Hurt MM, Brown PO, Botstein D (2002) Identification of genes periodically expressed in the human cell cycle and their expression in tumors. Mol Biol Cell 13:1977-2000 